\title{
Effect of Structural Fluctuations on Elastic Lifetimes of Adsorbate States - Isonicotinic Acid on Rutile(110)
}

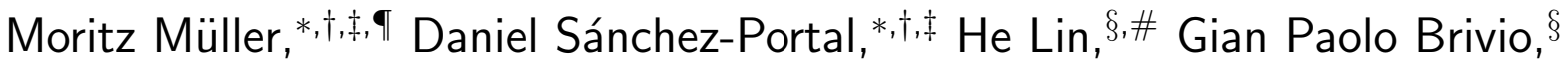 \\ Annabella Selloni, $\|$ and Guido Fratesi*, ${ }^{*}$ \\ †Centro de Fúsica de Materiales CSIC-UPV/EHU, Paseo Manuel de Lardizabal, 5, San \\ Sebastián, 20018, Spain \\ $\ddagger$ Donostia International Physics Center, Paseo Manuel de Lardizabal, 4, San Sebastián, \\ 20018, Spain \\ ฯ CIC nanoGUNE, Tolosa Hiribidea, 76, San Sebastián, 20018, Spain \\ $\S$ Dipartimento di Scienza dei Materiali, Università degli Studi di Milano-Bicocca, Via \\ Cozzi, 55, Milano, 20125, Italy \\ ||Department of Chemistry, Princeton University, Princeton, New Jersey, 08544, United \\ States \\ $\perp$ Dipartimento di Fisica, Università degli Studi di Milano, Via Celoria, 16, Milano, 20133, \\ Italy \\ \#Current address: Institute of Applied Chemistry, Xinjiang University, Urumqi, 830046 \\ Xinjiang, P. R. China \\ E-mail: mllr.mrtz@gmail.com; daniel.sanchez@ehu.eus; guido.fratesi@unimi.it
}

\begin{abstract}
We sample ab-initio molecular dynamics trajectories to address the impact of structural fluctuations on elastic lifetimes of adsorbate states at room temperature focusing on heterogeneous charge injection from isonicotinic acid as a key anchoring unit in dye-sensitized energy devices. Complementing related theoretical studies, we employ a Green's function technique based on density functional theory to account for a fully semi-infinite substrate of rutile $\mathrm{TiO}_{2}(110)$. We address the effect of a core-excitation enabling direct comparison with soft X-ray experiments. We find that room temperature fluctuations drastically improve the agreement with experimental lifetime measurements while the core-hole plays an important role shifting the spectra and reducing the electron vibrational coupling of the adsorbate states. Ultimately, the emerging resonance spectra highlight the role of the continuum of acceptor states in temperature broadened Voigt-type profiles.
\end{abstract}

\section{Introduction}

Ultrafast electronic processes at surfaces are at the heart of many applications in photochemistry, catalysis, and molecular electronics. After an initial excitation by light, ultrafast electron transfer takes place during generation of solar fuel, ${ }^{1}$ as well as from photo-excited dyes towards semiconducting substrates in dyesensitized solar cells (DSSCs). ${ }^{2-4}$ Although the original design of the DSSC has been challenged in recent years by perovskite-based solar cells, ${ }^{5,6}$ electronic charge injection remains the basic physical process behind these sunlight 
harvesting energy devices.

Among a variety of candidates, isonicotinic acid is a common anchoring unit in DSSCs as, for instance, in the high-performing N719-dye. ${ }^{7}$ Isonicotinic acid has been investigated extensively, from an experimental and theoretical point of view. In particular many studies have examined isonicotinic acid on different facets and structural phases of $\mathrm{TiO}_{2}$, which is the preferred material for the anode of DSSCs due to its stability, high mobility, mesoporous structure and high availability.

A series of core-spectroscopy studies by Schnadt et $a .^{8-12}$ investigated the structural and electronic properties of monoand multilayers of isonicotinic acid on rutile $\mathrm{TiO}_{2}(110)$. Using core-hole-clock spectroscopy, ${ }^{13-15}$ they extracted electron injection times from core-excited isonicotinic acid (identified as $\mathrm{LUMO}+2$ ) into the substrate at an ultrafast time-scale below 5 fs at monolayer coverage. ${ }^{8}$ The employed core-hole-clock technique can be pushed to measure charge-transfer times in the attosecond domain. ${ }^{16}$

From a theoretical point of view, the calculation of charge-transfer times from adsorbates has been approached using different techniques. ${ }^{17-19}$ For example, starting from standard density functional theory (DFT) calculations, Fermi's golden rule was used to estimate charge-transfer rates from the computed Hamiltonian matrix elements between the extended states in the substrate and the localized states in the adsorbate. In principle, the relatively short range of these interactions (proportional to the overlaps between adsorbate and substrate wavefunctions) can be exploited to design efficient algorithms to screen dyes for optimal charge injection ${ }^{17}$ as carried out by Martsinovich et al. ${ }^{20}$ These authors estimated lifetimes below 1 fs for isonicotinic acid in its equilibrium geometry (no core-excitation included) on rutile (110) and on anatase (101). ${ }^{20}$

While the calculations described above analyze static structures, some groups have already tried to incorporate the effect of temperature on the electron injection process. This has been done at different levels of theory. For example, Prezhdo et al. ${ }^{21-23}$ have used a combina- tion of non-adiabatic molecular dynamics based on fewest switches surface hopping ${ }^{24}$ and DFT. Employing this technique they found for the lowest unoccupied molecular orbital (LUMO) of isonicotinic acid on rutile (110) at $50 \mathrm{~K}$ a lifetime of 28 fs averaging over 10 different trajectories. ${ }^{25}$ In a subsequent work using a derivative of isonicotinic acid functionalized with silver cyanide, they found that the lifetime of the LUMO dropped to 5 fs when the temperature was increased to $350 \mathrm{~K}$ and 100 initial configurations were considered. Although it is difficult to obtain a precise picture of the effect of temperature from these simulations, since the data are not directly comparable (different derivatives and statistics are used), the authors make the interesting observation that the increased transfer rate is mostly due to adiabatic effects.

Batista et $a l .{ }^{26,27}$ studied the effect of thermal fluctuations, focusing on the case of catechol on anatase (101). They used ab initio molecular dynamics to generate trajectories at a given temperature in combination with extended Hückel theory to model the electron dynamics along the trajectory. They found lifetimes of the LUMO around 38 fs at $0 \mathrm{~K},{ }^{26}$ while an ensemble average of fixed initial configurations from a trajectory at $300 \mathrm{~K}$ reduces the injection time to about 6 fs. ${ }^{26}$ Additional non-adiabatic effects due to nuclear motion during electronic decay lead to further reduction of the lifetime to about 2.5 fs. ${ }^{27}$ This observation seems consistent with the one mentioned above concerning the relative importance of adiabatic and non-adiabatic effects. After the ultrafast initial injection to an interfacial charge state, Batista et al. also followed the delocalization of the electron into extended substrate states.

The approaches mentioned so far treat the ions as classical particles, which is usually necessary for such complex systems. However, there have been model calculations that use a full quantum treatment to study electron dynamics at the DSSC interface. ${ }^{28-31}$

Along similar lines, in this work, we investigate the elastic lifetimes of the frontier orbitals of isonicotinic acid on rutile $\mathrm{TiO}_{2}(110)$ in the presence of structural fluctuations by sampling static nuclear configurations from $a b$ - 
initio molecular dynamics at room temperature. ${ }^{32}$ Thereby we extended previous work considering the equilibrium structure $(0 \mathrm{~K})^{19}$ to include the effect of temperature. This approach does not include non-adiabatic effects explicitly. However, the sampling of the configurational space at a given temperature is included. Such sampling has been identified as a key ingredient to determine the temperature dependence of charge-transfer rates. The adopted Green's function technique improves the description of the electronic structure of the substrate over that provided by finite slabs and cluster models. ${ }^{18,19}$ Thus, our method is suitable to describe charge-transfer into fully delocalized states in the substrate. Additionally, and importantly, we include a core-hole in our simulations to obtain charge-transfer rates comparable with core-hole-clock experiments by Schnadt et al. ${ }^{8}$

We performed a detailed analysis of the cumulative theoretical spectra. Our results indicate that the inhomogeneous broadening due to the fluctuating energy positions of the resonances is not sufficient to explain their shapes. It is crucial to take into account the variation of the average elastic width with energy. The latter reflects the energy dependence of the density of states in the substrate.

The results section in this paper is organized in two parts, the first considering the adsorbate in its electronic ground-state and the second the core-excited case.

\section{Computational Methods}

In this work we analyze the effect of thermal structural fluctuations on the elastic linewidths of the molecular resonances. We access a set of structures representative of the configurational space sampled by the adsorbed molecule at finite temperature. Extending our previous study for the equilibrium configuration, we consider a number of snapshots generated along a previously calculated Car-Parinello (CPMD) trajectory ${ }^{32}$ of isonicotinic acid $\left(\mathrm{C}_{5} \mathrm{H}_{4} \mathrm{NCOOH}\right)$ adsorbed on rutile (110). The CPMD trajectory was computed at a constant temperature of 300K using a Nosé thermostat, a slab containing four $\mathrm{TiO}_{2}$ trilayers, a $3 \times 2 \mathrm{TiO}_{2}$ supercell in the lateral directions and one molecule per supercell adsorbed on one of the surfaces of the slab. The trajectory covers $10 \mathrm{ps}$ after an initial equilibration period of 2 ps. As described in detail in the Supporting Information, we devise a matching procedure that allows us to model an infinitely extended $\mathrm{TiO}_{2}$ substrate starting from the finite-slab CPMD geometries.

In the present work we have selected a total of 161 snapshots (at a constant sampling rate of one frame every $62.5 \mathrm{fs}$ ) and recomputed electronic structure of each configuration using the SIESTA/TranSIESTA package. ${ }^{33}$ As a first step, for each original structure we generated a new structure employing the above mentioned matching procedure (described in the Supporting Information). The idea is to construct geometries with a larger number of layers and approaching the perfect bulk phase inside the material. This is necessary to provide a smooth connection with the self-energies that describe the semi-infinite $\mathrm{TiO}_{2}$ substrate in our Green's function technique. In the final geometries only the two outermost trilayers of $\mathrm{TiO}_{2}$ and the adsorbate move according to the original CPMD dynamics. Hence, the third trilayer provides a smooth transition to the fixed bulk configuration, that is assumed in the inner layers. To cancel spurious dipoles the final slab is symmetrical and contains $9 \mathrm{TiO}_{2}$ trilayers in total. Periodic images are separated by at least $24.4 \AA$ of vacuum in order to avoid interaction between replicas. This setup is illustrated schematically in Fig. 1a, while Fig. 1c/d shows the details at the organic-semiconductor interface.

Our SIESTA calculations utilized a double$\zeta$ polarized basis set of numerical atomic orbitals $^{34}$ to expand the wavefunctions, whose radii were determined using a $100 \mathrm{meV}$ energy shift. ${ }^{33}$ The exchange-correlation functional by Perdew, Burke and Ernzerhof ${ }^{35}$ was employed in combination with norm-conserving TroullierMartins pseudopotentials. ${ }^{36}$ We used $3 \times 2 \times 1$ k-point sampling and a mesh cutoff value of $250 \mathrm{Ry}$, respectively, for the reciprocal and realspace grids. A tolerance of $10^{-4}$ was used for the changes of the density matrix in order to 
stop the self-consistent cycle.
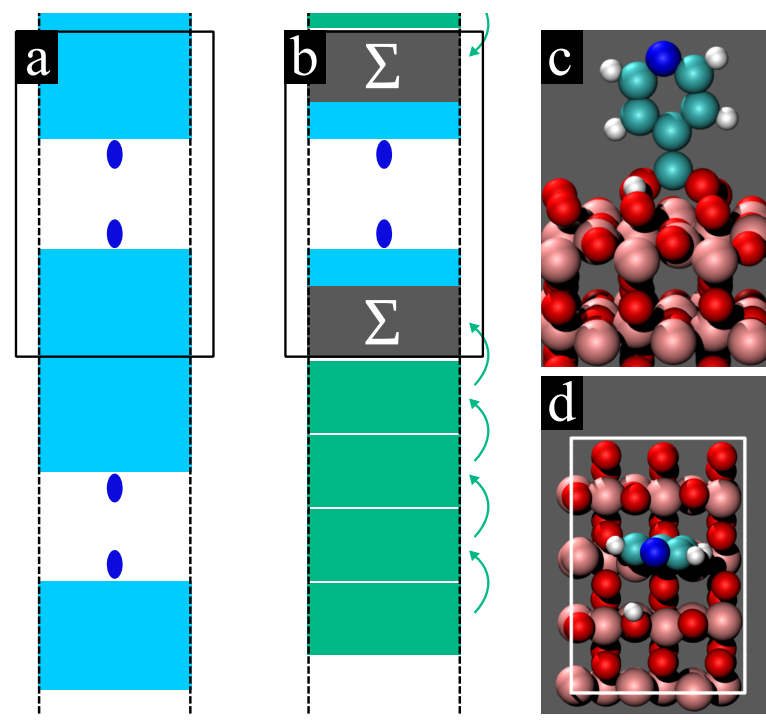

Figure 1: (a) As described in the text, a symmetric slab containing nine $\mathrm{TiO}_{2}$ trilayers plus one adsorbate in each surface is constructed starting from selected snapshots of the CPMD simulation in Ref. 32. (b) The coupling of the structure to a semi-infinite bulk support is included in the Green's function of the central region via self-energies $\Sigma$. Blue ovals represent adsorbates, light blue areas stem from finite slab calculations, while green areas refer to bulk calculations. The self-energies due to the bulk are added to the slab in the $\Sigma$-regions. The coupling of the adsorbed isonicotinic acid to the continuum of states in rutile(110) yields then the lifetime broadening. (c) Side view and (d) top view of the system. The black and white boxes refer to the computational unit cell.

In a final step we include the coupling of the scattering region (enclosing the decorated surfaces) to the fully semi-infinite $\mathrm{TiO}_{2}$ support (Fig. 1b) in an embedding approach. ${ }^{37-39}$ The coupling is accurately simulated inside the scattering region by adding self-energies $(\Sigma)$ due to the semi-infinite substrate (Fig. 1b) that connect to four trilayers (layers 3 to 6 ) of the initially calculated nine-trilayer slab. We use the Green's function procedure implemented in the TranSIESTA transport code. ${ }^{40}$ The $\mathrm{TiO}_{2}$ electrode was defined using $1 \times 2 \times 4$ bulk unit cells with a k-point sampling of $9 \times 2 \times 2$. To compute the Green's function an imaginary part of $\eta=0.02 \mathrm{eV}$ was added to the energies.

The Hamiltonian and self-energy matrices coming from bulk calculations (green areas Fig. 1b) and slab calculations (light blue areas, Fig. 1a) were aligned at a common reference to correctly include the coupling to bulk $\mathrm{TiO}_{2}$ in the Green's function procedure. This is important, since the Fermi level commonly used to align transport calculations for metallic systems cannot be used for calculations of semiconducting materials, which produce in practice arbitrary positions of the Fermi level inside the electronic gap. Additionally, the zero of potential in periodic systems is known to be arbitrary. ${ }^{41}$ To overcome both issues, the energy levels of bulk and slab calculations were aligned at the Hartree potential of the bulk $\mathrm{TiO}_{2}$ assuming that the bulk value of the Hartree potential is reached deep inside the slabs. We determine the macroscopically averaged Hartree potentials applying the smoothing method of Junquera et al. ${ }^{42,43}$ in the direction perpendicular to the surface. Subsequently, the two values are aligned at the transition towards the bulk region, making the bulk density of states (DOS) a common energy reference for all calculations. Such a common reference also enables the comparison of peak positions from different snapshots. For plotting purposes, the origin of the spectra was shifted to the valence band maximum (VBM) of bulk $\mathrm{TiO}_{2}$. This appeared to be a reasonable choice since the total DOS of the 9 trilayers and symmetrically attached adsorbates only showed small fluctuations of the VBM across different snapshots of the CPMD simulation.

We extract (resonant) elastic lifetimes of initial wave-packets derived from single molecular orbitals (MOs) of isonicotinic acid following the procedure in Refs. 18,44 and extensions to MOs in Refs. 19,45. For the calculation of the MOs the hydrogen atom dissociated upon adsorption is added back to the molecule in order to saturate free bonds. The reference wave-packet $\Phi$ is then obtained by setting the basis set coefficients related to the added hydrogen to zero and normalizing. This is expected to give good results as the coefficients for the hydrogen atom's orbitals are negligible for the MOs of interest 
here. ${ }^{19}$ We subsequently calculate the projected density of states (PDOS) on the reference wavepackets:

$$
\begin{aligned}
\rho_{\Phi}(E) & =\operatorname{Im}\left[\iint \Phi^{*}(\mathbf{r}) G\left(E, \mathbf{r}, \mathbf{r}^{\prime}\right) \Phi\left(\mathbf{r}^{\prime}\right) d \mathbf{r} d \mathbf{r}^{\prime}\right] \\
& \sim \frac{1}{\pi} \frac{\Omega}{\left(E-E_{R}\right)^{2}+\Omega^{2}},
\end{aligned}
$$

where $G\left(E, \mathbf{r}, \mathbf{r}^{\prime}\right)$ is the Green's function of the scattering region. The resulting spectra are fitted in an energy window of $\pm 0.8 \mathrm{eV}$ around their maxima by a Lorentzian function with a proportionality coefficient, and the resonance energy $E_{R}$ and the full width at half maximum of $2 \Omega$ as fitting parameters. The elastic linewidth $\Gamma$ of the resonance is extracted as $\Gamma=2(\Omega-\eta)$ considering that the Green's function was calculated at an imaginary energy $E+i \eta$. Employing the uncertainty relation we obtain the lifetime $\hbar / \Gamma(\hbar=658.21 \mathrm{fs} \cdot \mathrm{meV}$ in the relevant units).

In CPMD simulations ionic and electronic degrees of freedom are strictly separated due to the Born-Oppenheimer approximation. Nonadiabatic effects such as electronic transitions caused by ionic movement are hence explicitly excluded. As a result the electronic properties are governed by the electronic structure at every instance of time. In that spirit, considering a Lorentzian line shape as in Eq. (1) implies that the populations of the instantaneous initial states $P(t)$ decay exponentially with time. The dependence on temperature is then included in the statistical ensemble $\langle P(t)\rangle=$ $\langle\exp (-\Gamma t / \hbar)\rangle$, where $\langle\ldots\rangle$ denotes the arithmetic average over all snapshots. To simplify the forthcoming discussion, unless otherwise stated we approximate $\langle P(t)\rangle \approx \exp (-\langle\Gamma\rangle t / \hbar)$, where $\hbar /\langle\Gamma\rangle$ plays the role of an approximate average lifetime. The limit to this approximation is discussed at last.

To compare our simulations with core spectroscopy experiments, we effectively introduce a core-hole in our calculations by reducing the occupation of the 1s state of the nitrogen atom during the construction of the pseudopotential. In the slab calculations this core-hole is neu- tralized by adding back the removed electron to the valence. The model represents a metastable situation with a strongly localized hole on the nitrogen 1s level, and an electron has been excited into a molecular resonance. The transfer of this electron into the $\mathrm{TiO}_{2}$ support is the main process whose time-scale we aim to study.

\section{Elastic lifetimes in the elec- tronic ground state}

We start by analyzing isonicotinic acid adsorbed on rutile (110) in its electronic ground state. The corresponding spectra computed according to Eq. (1) are presented in Fig. 2. To investigate the dependency of the elastic linewidths on the adsorption and molecular geometry we calculated the projections of the Kohn-Sham density of states (DOS) onto the adsorbate states ranging from the HOMO-1 to the $\mathrm{LUMO}+2$ at different snapshots along a CPMD simulation at a constant temperature of $300 \mathrm{~K}$, as well as in the equilibrium geometry. The latter case is shown in Fig. 2a, and Table 1 presents the corresponding extracted linewidths $\Gamma_{e q}$ and peak positions of the molecular resonances $E_{R}$. These values are in overall good agreement with previously reported values by Fratesi et al. 19 (shown in brackets). The observed deviations are attributed to the larger unit-cell in the present calculations $(3 \times 2$ instead of $3 \times 1)$. Another difference with respect to the model in Ref. 19 lies in the chosen adsorption site for the dissociated hydrogen, that is put closer to the molecule in the current work, following the CPMD study. ${ }^{32}$ However, we explicitly checked that this choice has negligible effect on the reported results.

We now compare the case of the equilibrium structure with instantaneous snapshots from our CPMD simulation. We sample the 10 ps trajectory taking 161 snapshots after an initial equilibration time of 2 ps. Under the effect of temperature the resonance peaks move around their equilibrium positions and relative to the DOS of the bulk $\mathrm{TiO}_{2}$ (Fig. 2b). The overall picture suggests a correlated motion of the energy position of the different levels across time 
Table 1: Computed values of the linewidths $\Gamma_{e q}$, associated lifetimes $\hbar / \Gamma_{e q}$, and resonance positions $E_{R}$ of the molecular states at the equilibrium adsorption geometry. These values are to be compared with the corresponding averages obtained using the structures visited during the CPMD trajectory: Mean energy positions of the molecular resonances $\left\langle E_{R}\right\rangle$ and their standard deviations $\sigma\left(E_{R}\right)$, average linewidths $\langle\Gamma\rangle$ and their relative standard error $\delta(\langle\Gamma\rangle)$, lifetimes $\hbar /\langle\Gamma\rangle$. The values for the equilibrium adsorption shown in brackets are taken from Ref. 19. All quantities are further defined in the text. All energies are referred to the $\mathrm{VBM}$ of $\mathrm{TiO}_{2}$.

\begin{tabular}{|c|ccc|ccccc|}
\hline & \multicolumn{3}{|c|}{ equilibrium $(0 \mathrm{~K})$} & \multicolumn{4}{c|}{ thermal average $(300 \mathrm{~K})$} \\
\cline { 2 - 8 } & $E_{R}$ & $\Gamma_{e q}$ & $\hbar / \Gamma_{e q}$ & $\left\langle E_{R}\right\rangle$ & $\sigma\left(E_{R}\right)$ & $\langle\Gamma\rangle$ & $\delta(\langle\Gamma\rangle)$ & $\hbar /\langle\Gamma\rangle$ \\
& $(\mathrm{eV})$ & $(\mathrm{meV})$ & $(\mathrm{fs})$ & $(\mathrm{eV})$ & $(\mathrm{eV})$ & $(\mathrm{meV})$ & $(\%)$ & $(\mathrm{fs})$ \\
\hline HOMO & 0.27 & $0(0)$ & $\infty(\infty)$ & 0.27 & 0.45 & 11 & 43 & 62 \\
LUMO & 3.53 & $218(186)$ & $3(4)$ & 3.47 & 0.30 & 280 & 5 & 2 \\
LUMO+1 & 4.61 & $1(0)$ & $657(\infty)$ & 4.61 & 0.27 & 6 & 10 & 105 \\
LUMO+2 & 5.93 & $35(15)$ & $19(44)$ & 5.83 & 0.29 & 57 & 5 & 12 \\
\hline
\end{tabular}

(peak ordering and distances between peaks tend to keep constant), although deviations from a rigid shift can be observed. In Fig. $2 b$ it is visible that the HOMO lies within the band gap for most of the snapshots. Just in a few cases it is severely downshifted, which is most clearly seen in Fig. 3. The LUMO splits into two peaks corresponding to a bonding (lower energy peak) and an antibonding (higher energy peak) interaction with the substrate. ${ }^{19}$ This is particularly apparent for the equilibrium geometry as shown in Fig. 2a. For most of the snapshots the bonding component of the LUMO melts into an extended tail towards the edge of the conduction band of the $\mathrm{TiO}_{2}$ substrate and it does not give any distinct peak in the cumulative spectrum in Fig. 2c. Contributions to the spectrum from the antibonding part dominate the LUMO's PDOS, and the reported values in Table 1 and Fig. 3 correspond to this antibonding peak. The LUMO+1 is uncoupled from the substrate in the equilibrium geometry and structural fluctuations preserve the single peak shape, only causing a small increase in width. Peak broadening during the dynamics is also visible in the case of the $\mathrm{LUMO}+2$, where already at the equilibrium structure coupling to the substrate yields a finite width. Strong couplings to the substrate for distorted configurations induce additional deviations from the Lorentzian line-shape as visible in several snapshots in Fig. 2b.
In spectroscopy experiments probing the density of states under typical conditions, thermal averages of spectra are recorded. Even at zero temperature such spectra usually correspond to an ensemble of different structures, as the configuration may vary from molecule to molecule. In analogy to such experiments we average our set of calculated spectra from different snapshots. The resulting cumulative spectra are shown in Fig. 2c. While the finite sampling still leads to spiky features for the weakly interacting $\mathrm{HOMO}$ and $\mathrm{LUMO}+1$ states (reflecting the need of a larger statics), for the more strongly coupled LUMO and LUMO+2 the spectra approach a smooth profile. Regarding the LUMO resonance, its bonding component is smeared out in the cumulative spectrum reducing the possibility of a separate experimental measurement of its lifetime at room temperature, as was suggested for the zero temperature case. ${ }^{19} \mathrm{We}$ note that the width of the peaks in the cumulative spectrum cannot be directly related to electronic charge-transfer times, as the latter depend on the instantaneous electronic couplings, while the former are dominated by the fluctuations of the MO energies at room temperature. Hence, thermally broadened spectra do not provide direct access to electronic lifetimes. Instead, more sophisticated experimental techniques such as the core-hole-clock method or high resolution pump-probe experiments are required. 


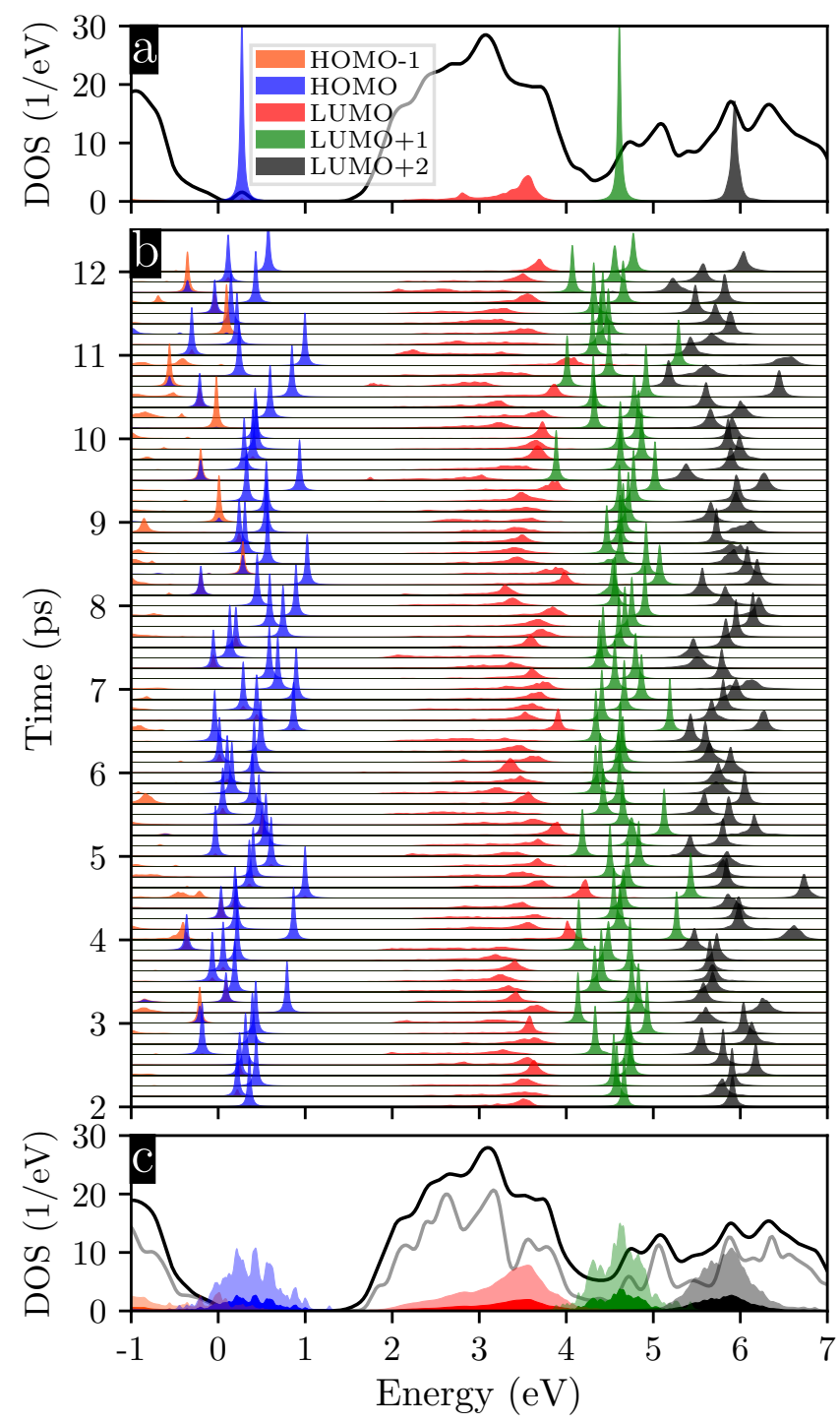

Figure 2: Density of states projected onto the frontier orbitals (HOMO-1 up to $\mathrm{LUMO}+2$ ) of isonicotinic acid (Eq. 1): (a) Spectrum for the equilibrium structure. (b) 81 snapshots during 10 ps of the CPMD at $300 \mathrm{~K}$ (every second snapshot of the full sampling shown). (c) $\mathrm{Cu}$ mulative spectra after averaging all 161 snapshots (colored areas), additionally repeated after rescaling $(\times 4)$ to facilitate visualization (light shaded areas). Black lines in (a) and (c) show the density of states (DOS) $(\times 0.14)$ from finite slab calculation. The gray line in (c) shows the bulk $\mathrm{TiO}_{2}$ DOS $(\times 0.6)$. The origin of all the spectra was shifted to the VBM of the bulk DOS as a common energy reference.

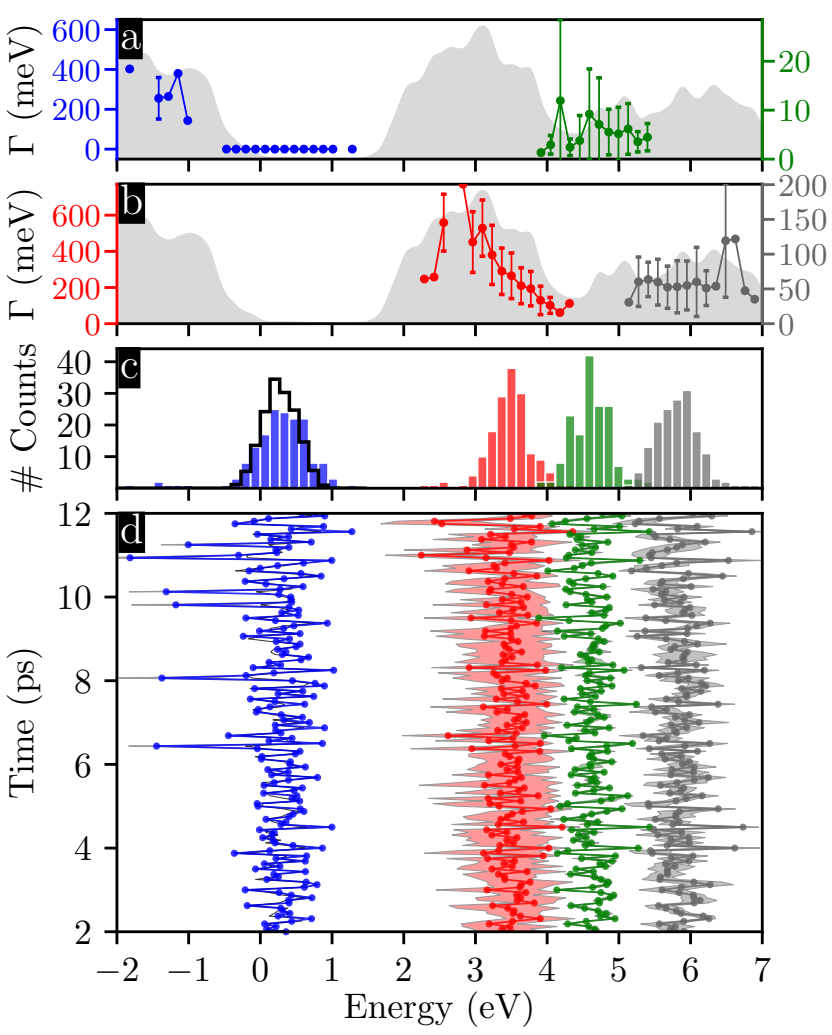

Figure 3: Fluctuations of the positions and widths of the molecular resonances for 161 snapshots of the CPMD trajectory. The values were extracted from a Lorentzian fit to the PDOS of the frontier orbitals of isonicotinic acid (cf. Fig. 2b): (d) Evolution of the peak positions. Shaded areas indicate the corresponding widths $(\sqrt{\Gamma}$ plotted instead of $\Gamma$ to make make small changes in width more visible) (c) Histograms of the resonance positions. $(a, b)$ Mean widths of the Lorentzian fits and their standard deviations (error bars) in each of the energy bins of the histograms in (c), highlighting the importance of the DOS in the substrate (light shaded areas). Two separate plots $(a, b)$ are presented to allow the use of different scales on the y-axes for each set of data (colored dots) and to avoid the overlapping of the data in energy. Black lines in $(\mathrm{c}, \mathrm{d})$ show the highest occupied state during CPMD trajectory (corresponds, in principle, to the HOMO) for which larger statistics regarding its energy position are available. 
For a quantitative statistical analysis, we examine the mean value $\left\langle E_{R}\right\rangle$ of the MO resonance positions $E_{R}$ and its standard deviations $\sigma\left(E_{R}\right)$. All extracted values are listed in Tab. 1. A binning procedure allows us to analyze the distribution of $E_{R}$ during the dynamics, as reported in Fig. 3c. The number of sampled configurations is sufficient to approximately reproduce a Gaussian shape of the distributions. As expected, the thermal averages $\left\langle E_{R}\right\rangle$ are found to be in close agreement with the peak positions in the equilibrium geometry $E_{R, e q}$. However, the spreads of the values of $E_{R}$ are relatively large, as evidenced both by the plot in Fig. 3c and by their standard deviations $\sigma\left(E_{R}\right)$ that reach about $0.3 \mathrm{eV}$ (even $0.45 \mathrm{eV}$ for the HOMO). Overall, the size of the fluctuations in our study is consistent with observations in related works. For example, Prezhdo and coworkers find slightly smaller energy fluctuations at $50 \mathrm{~K}$ for the evolution of the excited state (state with the highest occupation after initial filling of the Kohn-Sham LUMO) in non-adiabatic molecular dynamics simulations of isonicotinic acid on rutile (110). ${ }^{25}$ Furthermore, for a structurally strongly related compound (consisting of isonicotinic acid with an added $\mathrm{AgCN}$ group) on $\mathrm{TiO}_{2}$ they report an energy range of up to $1 \mathrm{eV}$ for the resonance position at $350 \mathrm{~K} .{ }^{46}$ Similarly, for alizarin on $\mathrm{TiO}_{2}$ they report a standard deviation of $0.39 \mathrm{eV}^{47,48}$ at ambient temperature.

To further assess the statistical distribution of the resonance positions $E_{R}$ we compare the results for the HOMO with those related to the highest occupied electronic state following its evolution during the CPMD simulation, where more than 5000 steps were recorded. A Gaussian-like distribution is even more apparent in the latter case, as can be seen from the histogram in Fig. 3c (to facilitate comparison to the other plots, its area is also normalized to $N=161$ ). It is interesting to see that the position and shape of both distributions is very similar. Indeed, we can see that the energies of the highest occupied level during the CPMD (computed using a finite slab and planewaves, thin black line in Fig. 3d) and the mean position of the HOMO projection of the sur- face Green's function (calculated using a semiinfinite surface and the SIESTA/TranSIESTA package, blue line in Fig. 3d) agree very well at most of the 161 snapshots selected. They only deviate in a few snapshots where the HOMO and the HOMO-1 projections cross (e.g., at 9 ps in Fig. 2b). This supports the reliability of the procedure (see Supporting Information) used to match the fluctuating structures of the adsorbate and surface layers (from the slab calculation) to the static semi-infinite bulk necessary to incorporate the effect of the substrate.

To quantitatively assess our theoretical results for elastic lifetimes, we extracted the linewidth of the MO peaks for each snapshot of Fig. $2 \mathrm{~b}$ by Lorentzian fitting as illustrated in the methods section. The average widths presented in Table 1 follow the trend $\left\langle\Gamma^{\mathrm{LUMO}+1}\right\rangle<$ $\left\langle\Gamma^{\mathrm{HOMO}}\right\rangle<\left\langle\Gamma^{\mathrm{LUMO}+2}\right\rangle<\left\langle\Gamma^{\mathrm{LUMO}}\right\rangle$, which is similar to that found for the equilibrium geometry apart from $\left\langle\Gamma_{\text {HOMO }}\right\rangle$. Unlike for the equilibrium structure, the HOMO state enters the valence band at several simulation times where it couples strongly to the substrate and we get a finite average electronic width of $\left\langle\Gamma_{\text {Номо }}\right\rangle \sim 11 \mathrm{meV}$ (i.e., an average lifetime $\hbar /\langle\Gamma\rangle \sim 62 \mathrm{fs})$. Also the linewidth of the other analyzed peaks increases in comparison to the equilibrium structure. For example, the LUMO+1 state is strongly broadened leading to a reduction of about half a picosecond in its charge transfer time. Overall the effect of the structural fluctuations clearly shows that it is indispensable to take into account an extended set of geometries in order to obtain a realistic picture of the charge transfer times in a device.

We stress that structural fluctuations not necessarily tend to increase the average linewidths of adsorbate levels, although we observed such behavior here. For example, the opposite effect may occur for a molecular resonance whose energy in the equilibrium geometry overlaps with a large peak of the DOS of the substrate. In that case, deviations of the peak position may result in a reduction of the average linewidth due to the reduced availability of final states in the substrate in the configurations visited during the dynamics.

The coupling to a continuum of states in the 
substrate is usually described by Fermi's golden rule 20,49

$$
\Gamma\left(E_{R}\right)=2 \pi\left|M_{R S}\left(E_{R}\right)\right|^{2} \rho_{S}\left(E_{R}\right),
$$

where $M_{R S}\left(E_{R}\right)$ is the Hamiltonian matrix element coupling the resonance $R$ to the electronic states $S$ in the substrate at a given energy $E_{R}$, and $\rho_{S}\left(E_{R}\right)$ is the density of states in the substrate. Here, by averaging $\Gamma$ over the various snapshots, we effectively deal with a thermally averaged version of this equation, $\left\langle\Gamma\left(E_{R}\right)\right\rangle=$ $2 \pi\left\langle\left|M_{R S}\left(E_{R}\right)\right|^{2} \rho_{S}\left(E_{R}\right)\right\rangle$. We note that the density of states $\rho_{S}\left(E_{R}\right)$ depends on the adsorption configuration only through the resonance energy $E_{R}$. However, the couplings $M_{R S}\left(E_{R}\right)$ are in general dependent on fine structural details and on $E_{R}$, since the character (e.g., symmetry, shape, decay into vacuum, ...) of the states from the substrate is also depending on energy. For a more intuitive understanding of the results it is desirable to separate the role of $\rho_{S}\left(E_{R}\right)$ and $\left|M_{R S}\left(E_{R}\right)\right|^{2}$. In particular, the above expression simplifies if both ingredients entering Eq. 2 behave as statistically independent, i.e., $\left\langle\left|M_{R S}\left(E_{R}\right)\right|^{2} \rho_{S}\left(E_{R}\right)\right\rangle \approx$ $\left\langle\left|M_{R S}\left(E_{R}\right)\right|^{2}\right\rangle\left\langle\rho_{S}\left(E_{R}\right)\right\rangle$, leading to

$$
\left\langle\Gamma\left(E_{R}\right)\right\rangle \approx 2 \pi\left\langle\left|M_{R S}\left(E_{R}\right)\right|^{2}\right\rangle\left\langle\rho_{S}\left(E_{R}\right)\right\rangle .
$$

The validity of this approximation in our specific case was investigated by evaluating separately $\left\langle\rho_{S}\left(E_{R}\right)\right\rangle$ and $\left\langle\left|M_{R S}\left(E_{R}\right)\right|^{2}\right\rangle$, operatively defined as $\left\langle\Gamma\left(E_{R}\right) /\left[2 \pi \rho_{S}\left(E_{R}\right)\right]\right\rangle$. The excellent agreement between the values of $\left\langle\Gamma\left(E_{R}\right)\right\rangle$ and of $2 \pi\left\langle\left|M_{R S}\left(E_{R}\right)\right|^{2}\right\rangle\left\langle\rho_{S}\left(E_{R}\right)\right\rangle$ (see Tables S1 and S2 in the Supporting Information) confirms their approximate statistical independence for isonicotinic acid on rutile $\mathrm{TiO}_{2}$. This indicates that the average character of the substrate states does not change dramatically within the energy windows explored by the resonances as the system fluctuates at room temperature and, thus, $M_{R S}\left(E_{R}\right)$ does not exhibit a strong variation as a function of $E_{R}$ (note that this is still compatible with a strong dependence on other variables, e.g. structural ones, which however do not exhibit a one-to-one correspondence with $\left.E_{R}\right)$. We are then enabled to disentangle the role of the matrix elements and the DOS, and to analyse the average coupling matrix $\left\langle\left|M_{R S}\right|^{2}\right\rangle$ as a physically meaningful quantity.

Additional insight into this aspect is provided by evaluating subset averages of $\Gamma$ within narrow energy intervals, for which we take the bins of Fig. 3c. The resulting $\langle\Gamma\rangle_{\text {bin }}$ values are shown in Figures 3a and b), where it is apparent that the average widths scale with the amount of available acceptor states. Thus, structural fluctuations at the interface affect the coupling matrix $\left|M_{R S}\right|^{2}$ in a way that is on average independent of the energy $E_{R}$ at which the molecular resonance appears. Nevertheless, fluctuations of the width $\Gamma$ of single snapshots even within the bins are relatively large as shown by their standard deviations (error bars in Figures 3a and $\mathrm{b}$ ).

To summarize the discussion of isonicotinic acid in its ground-state electronic configuration, we return to the cumulative spectra in Fig. 2c. Those spectra are the result of two broadening mechanisms. The electronic coupling leads to approximately Lorentzian-like peaks for the molecular resonances in each snapshot, with some exceptions to this behavior depending on the details of the electronic structure around the resonances and the strength of the coupling to the substrate. Within our classical treatment of the dynamics of ions, thermal fluctuations result in a Gaussian-like distribution of the resonance positions. Then, neglecting deviations from the two approximations described above, the cumulative spectra (see Fig. 2c) of the MO PDOS may be approached by a sum of Voigt curves, i.e., convolutions of Gaussians with Lorentzian profiles. Such Voigt or PseudoVoigt curves are routinely used to fit experimental spectra, ${ }^{50}$ where additional broadening due to the resolution of detector and broadening of incident beams must be also taken into account in order to extract relevant electronic couplings. A detailed discussion of such a fitting of the computed cumulative spectra with Voigt-type curves can be found in the Supporting Information. As described there, we find that the quality of the fit is improved if the energy dependence of the average elastic width is taken into account. This energy dependence de- 
scribes mostly the variation of the DOS of the substrate.

\section{Elastic lifetimes upon inclu- sion of the $\mathrm{N}$ 1s core-hole}

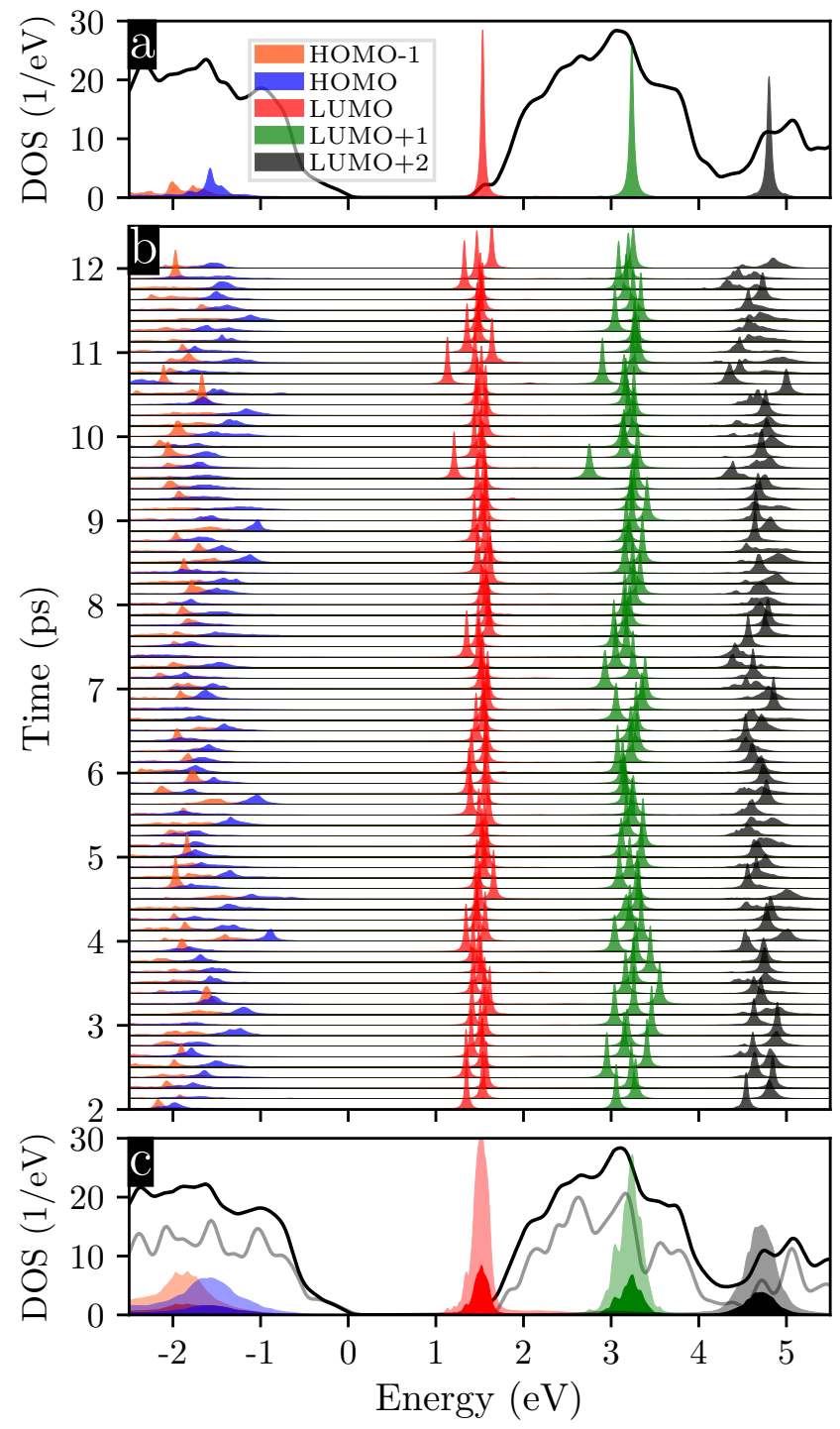

Figure 4: Spectra as in Fig. 2 but including a $\mathrm{N}$ 1s core-hole in the calculations.

In this section we include a core-hole in our calculations as necessary to quantitatively compare our theoretical charge-transfer times with available data from core-hole-clock experiments. ${ }^{8,19}$ The core excited (Fig. 4a) and the ground state systems (Fig. 2a), both computed in the ground-state equilibrium structure, exhibit striking differences since the levels shift downwards in energy due to the presence of the

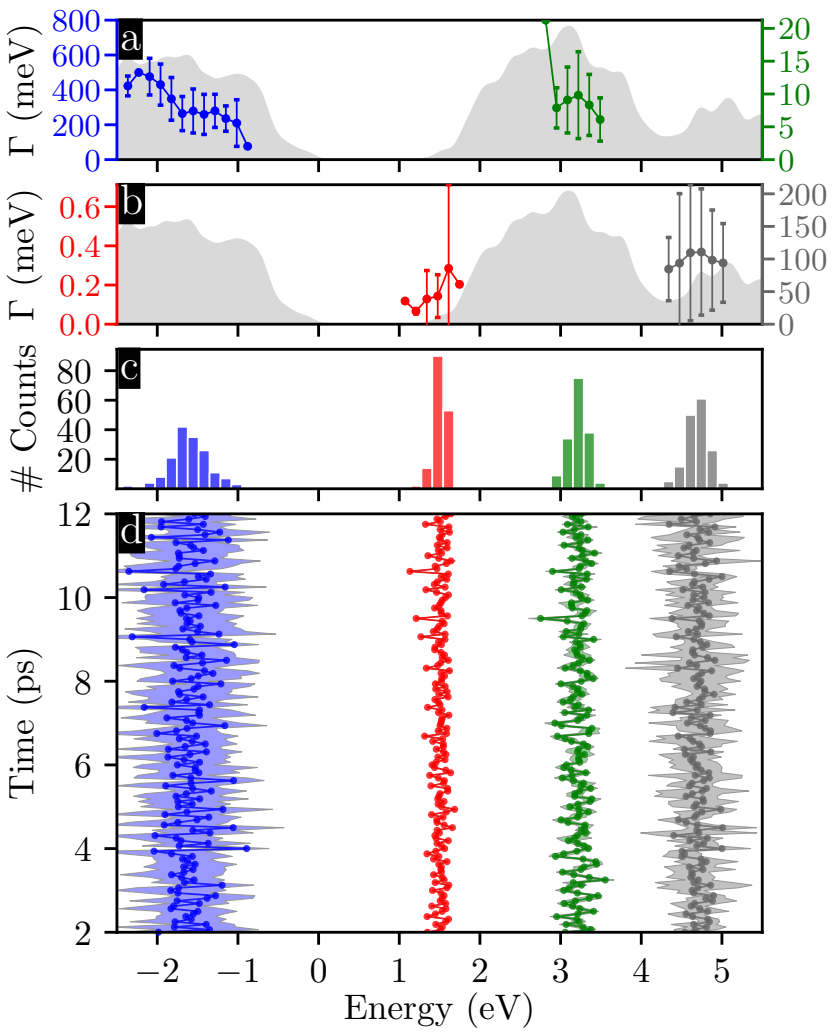

Figure 5: Energy positions of the resonances and associated elastic linewidths for the coreexcited case. The figure is organized analogously to Fig. 3. 
Table 2: Energy positions and linewidths of the electronic levels extracted from the spectra calculated for the core-excited molecule. The values presented in brackets are taken from Ref. 19 and were computed only at the equilibrium adsorption structure. The values in this Table can be contrasted with those presented in Table 1 for the molecule in its ground-state electronic configuration.

\begin{tabular}{|c|ccc|ccccc|}
\hline & \multicolumn{3}{|c|}{ equilibrium $(0 \mathrm{~K})$} & \multicolumn{4}{c|}{ thermal average $(300 \mathrm{~K})$} \\
\cline { 2 - 9 } & $E_{R}$ & $\Gamma_{e q}$ & $\hbar / \Gamma_{e q}$ & $\left\langle E_{R}\right\rangle$ & $\sigma\left(E_{R}\right)$ & $\langle\Gamma\rangle$ & $\delta(\langle\Gamma\rangle)$ & $\hbar /\langle\Gamma\rangle$ \\
& $(\mathrm{eV})$ & $(\mathrm{meV})$ & $(\mathrm{fs})$ & $(\mathrm{eV})$ & $(\mathrm{eV})$ & $(\mathrm{meV})$ & $(\%)$ & $(\mathrm{fs})$ \\
\hline HOMO & -1.57 & 152 & 4 & -1.60 & 0.24 & 292 & 3 & 2 \\
LUMO & 1.53 & 0 & $\infty$ & 1.51 & 0.08 & 0 & 11 & $\infty$ \\
LUMO+1 & 3.24 & $9(7)$ & $75(93)$ & 3.21 & 0.12 & 9 & 5 & 72 \\
LUMO+2 & 4.80 & $15(15)$ & $45(44)$ & 4.69 & 0.13 & 106 & 7 & 6 \\
\hline
\end{tabular}

positive core-charge. The HOMO level is now positioned inside the valence band of the substrate and consequently is strongly broadened so that it overlaps with the HOMO-1 level. Furthermore, the LUMO gets pinned to the edge of the conduction band, where the added electron (excited from the core $\mathrm{N}$ 1s level) gets localized and screens the positive charge of the Nitrogen core-hole. Although the LUMO+1 peak of the core-excited spectrum is located at energies comparable to those of the LUMO peak in the ground-state spectrum, the $\mathrm{LUMO}+1$ remains largely decoupled from the substrate. This is in contrast to the case of the LUMO of the unexcited molecule, where interaction with the substrate is favored by the symmetry and spatial distribution of the MO. ${ }^{19}$ Similarly, the width of the LUMO+2 peak in the core-excited system is smaller than in the ground-state case. The extracted values of the linewidths at the equilibrium geometry and the corresponding lifetimes can be found in Table 2, the values for the equilibrium adsorption structure from Ref. 19 are given in brackets. We find a good agreement between the two sets of data. As pointed out by Fratesi et al. ${ }^{19}$ coincidentally the energy level alignment of the adsorbate and substrate states compare well with those reported experimentally. ${ }^{8}$ This can be considered as a fortuitous cancellation of errors in this specific case, given that semi-local DFT functionals tend to misdiagnose the gaps of semi-conductors and the HOMO-LUMO gaps of adsorbates, as well as their relative level alignment.

We now turn towards investigating the effect of structural fluctuations in the presence of the core-hole, paralleling our previous discussion for the ground state case. We discuss the evolution of the spectra along the CPMD trajectory (Fig. 4b), in comparison to the spectrum at the equilibrium structure (Fig. 4a), and address the cumulative spectrum (Fig. 4c) including the effect of the finite temperature fluctuations. The shift of the molecular levels to lower energies due to the presence of the core-hole is similar for all the snapshots along the CPMD trajectory (Fig. 4b). The HOMO-1 and the HOMO level are strongly hybridized with the substrate and show overlapping distributions in the density of states. In a few simulation snapshots these two resonances exchange their relative energy positions. The situation is different for the LUMO and LUMO+1 states showing only a weak coupling to the surface during the whole CPMD simulation. This is particularly true in the case of the LUMO state spending most of its time inside the substrate's gap. Thus, these peaks keep a predominantly Lorentzian shape in the successive spectra displayed in Fig. 4b. In contrast, the LUMO+2 is found to hybridize strongly with the substrate in many snapshots, to the extent that the resonance splits into more than one peak in some cases. This is also in contrast to the case of the ground-state equilibrium structure, for which the LUMO+2 state of the core-excited molecule is more weakly coupled than in most of the CPMD snapshots (compare the values of $\Gamma_{e q}$ and $\langle\Gamma\rangle$ in Table 2$)$.

For the LUMO, LUMO+1 and LUMO+2 we observed considerably smaller fluctuations of 
the peak positions along the CPMD trajectory than in the ground state. This is reflected by smaller values for $\sigma\left(E_{R}\right)$ in Table 2 as compared to those in Table 1. As a consequence of this and the moderate coupling to the substrate, we find well separated peaks for each of these resonances in the cumulative spectra in Fig. 4c. This clearly differs from the ground-state case shown in Fig. 2c.

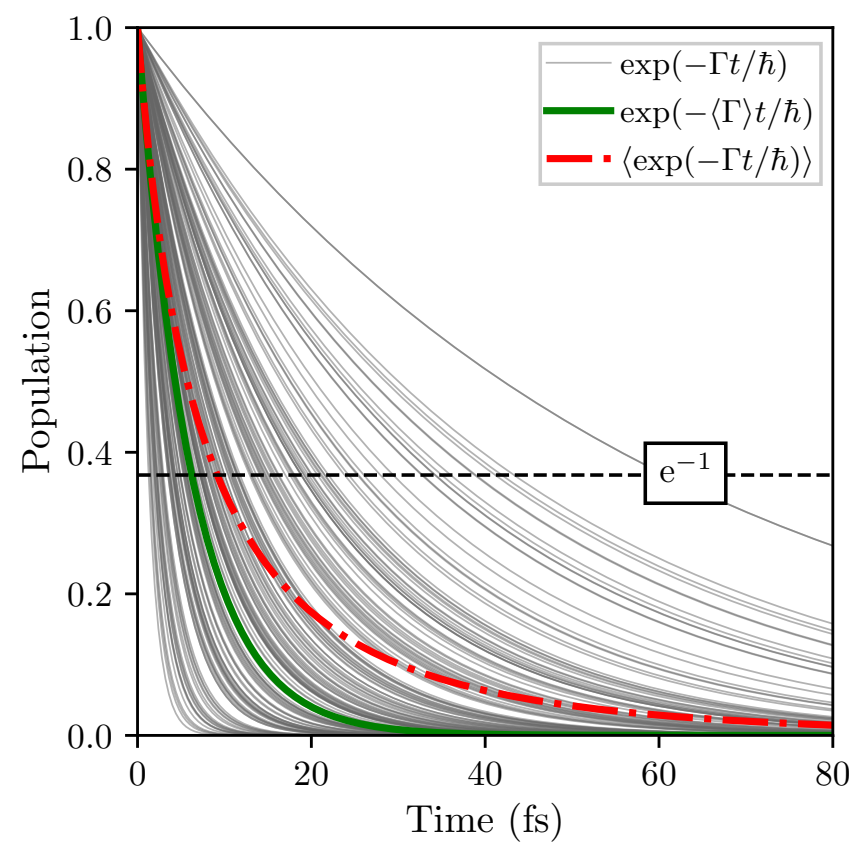

Figure 6: Exponential decays corresponding to the calculated $\mathrm{LUMO}+2$ linewidths $\Gamma$ for the sampled snapshots during the CPMD trajectory (gray lines). The lifetime $\tau$ of the state, defined as the time to decay to a population of $1 / e$, is slightly increased when considering the average of the exponential decays (broken red line) instead of the exponential determined by the the averaged linewidth $\langle\Gamma\rangle$ (thick green line).

As in the section covering the electronic ground-state configuration, we carry out a quantitative analysis by extracting the maximum peak positions $E_{R}$ and the width $\Gamma$ (using Lorentzian fitting) for a large number of snapshots during the CPMD simulation. The data is visualized in Fig. 5d and the corresponding values summarized in Tab. 2. The scaled widths displayed in the plot Fig. $5 \mathrm{~d}$ show the trend $\left\langle\Gamma^{\mathrm{LUMO}}\right\rangle<\left\langle\Gamma^{\mathrm{LUMO}+1}\right\rangle<\left\langle\Gamma^{\mathrm{LUMO}+2}\right\rangle<$ $\left\langle\Gamma^{\mathrm{HOMO}}\right\rangle$, which coincides with the trend for the equilibrium geometry (see Tab. 2). Regardless of the finite temperature fluctuations, the LUMO maintains a vanishing $(<1 \mathrm{meV})$ width and is approximately pinned to the conduction band edge. The coupling to the substrate of the $\mathrm{LUMO}+1$ resonance is similar during the CPMD steps than for the equilibrium structure. Indeed, the average $\left\langle\Gamma^{\mathrm{LUMO}+1}\right\rangle$ equals $\Gamma_{e q}^{\mathrm{LUMO}+1}$, although the estimated relative error of $\delta(\langle\Gamma\rangle)=\sigma(\Gamma) /(\langle\Gamma\rangle \sqrt{N})=5 \%$ for $N=161$ snapshots (sample size) indicates that the width slightly fluctuates along the trajectory $(\sigma(\Gamma)$ refers to the standard deviation of the linewidths). The average linewidths of the $\mathrm{HOMO}$, LUMO, and $\mathrm{LUMO}+1$ states retain the same order of magnitude when including the influence of fluctuations. Conversely, the $\mathrm{LUMO}+2$ peak is significantly broadened from $\Gamma_{e q}^{\mathrm{LUMO}+2}=15 \mathrm{meV}$ to $\left\langle\Gamma^{\mathrm{LUMO}+2}\right\rangle=110 \mathrm{meV}$. Correspondingly, its lifetime decreases from $\hbar / \Gamma_{e q}=45$ fs to $\hbar /\langle\Gamma\rangle=6$ fs, to be compared to an experimental value of $5 \mathrm{fs}^{8}$ Therefore, the inclusion of structural fluctuations improves considerably the agreement to available experimental data.

In Fig. 6 we explore two possible definitions of the mean lifetime of an electronic state under the effect of structural fluctuations. So far we have identified the average lifetime $\tau$ in connection with the average elastic linewidth, i.e., giving rise to the exponential decay of the molecular orbital occupation $\exp (-\langle\Gamma\rangle t / \hbar)$. A reasonable alternative would be to extract the elastic lifetime from the average of the exponential decays for all the snapshots along the molecular dynamics, $\langle\exp (-\Gamma t / \hbar)\rangle$. The lifetime would then be defined as the solution of the equation $\langle\exp (-\Gamma t / \hbar)\rangle=\exp (-1)$, which we solve graphically in Fig. 6 for the LUMO+2 resonance. We find that such alternative analysis produces larger lifetimes, although the difference is moderate and it does not change the results qualitatively. For example, in the case of the LUMO+2 this increases the computed elastic lifetime from 6 fs to 10 fs, thus retaining the improved agreement with the experiment.

The observation that, for core-excited isonicotinic acid on $\mathrm{TiO}_{2}$, only the $\mathrm{LUMO}+2$ resonance features a significant increase in width 
upon inclusion of structural fluctuations can be rationalized in terms of the symmetry and spatial distribution of the MOs. Only the LUMO and the $\mathrm{LUMO}+2$ state exhibit a significant overlap with the substrate ( $\pi$-type bonding visible in Fig. S7) and, thus, making the coupling very sensitive to interfacial structural fluctuations. However, the LUMO appears in an energy position with a low density of acceptor states (spending a large amount of time inside the substrate gap), resulting in the quenching of the corresponding decay channel ${ }^{8}$ and leaving the elastic width of the $\mathrm{LUMO}+2$ resonance as the only one largely affected by the dynamics.

Analogously to the ground state case we analyze the distributions of the resonance positions of the MOs and compare their standard deviations during dynamics. The respective values in Tab. 2 show in general a significant decrease of the standard deviations $\sigma\left(E_{R}\right)$ of the unoccupied state's energy upon inclusion of the corehole, with respect to the case without the corehole in Tab. 1. Most significantly the fluctuations of the LUMO's energy level are decreased upon pinning to the conduction band edge leading to a low standard deviation of $0.08 \mathrm{eV}$. Approximately reduced by a factor of two appear the fluctuations of the HOMO, the LUMO+1, and the $\mathrm{LUMO}+2$ which have standard deviations of $0.24 \mathrm{eV}, 0.12 \mathrm{eV}$, and $0.13 \mathrm{eV}$, respectively. This reduction of the temperature broadening can be rationalized as a result of the localization of the adsorbate states on isonicotinic acid due to the attractive interaction with the core-hole, making them less susceptible to the structural fluctuations at the surfacemolecule interface and to low energy fluctuations of the molecular structure.

Fig. 5 analyzes the distribution of the resonance positions and widths over energy. Consistent with the observations made in the previous paragraph, the distributions of both the $\mathrm{LUMO}+1$ and LUMO+2 states can be described by narrow Gaussian-like curves (see Fig. 5c). In contrast, HOMO and LUMO states do not present such symmetric energy distributions exhibiting tails towards the band edges. Similarly to the ground-state case, we find that also in the excited state the average widths of the peaks referring to each of the energy bins in Fig. 5c follow approximately the DOS of the substrate as shown in Fig. 5a and b. This suggests that the energy dependence of the DOS plays a key role in determining the average coupling of each resonance and the shape of the cumulative spectra in Fig. 4c.

\section{Conclusions}

In this work we theoretically investigated ultrafast electron injection from a prototype dye to the rutile $\mathrm{TiO}_{2}(110)$ substrate where it is adsorbed. We used density functional theory slab calculations to describe the dynamics of the molecule at room temperature and Green's function techniques to compute the resonant lifetimes of the molecular states. We sampled our 10 ps Car-Parinello molecular dynamics trajectory with 161 snapshots, extending related earlier studies on this system ${ }^{19,20,25}$ to include the effect of thermal fluctuations.

In addition to the molecular ground state electronic configuration, here we explicitly considered an excited case with a full core-hole in the 1s shell of Nitrogen. This is instrumental to compare to existing core-hole-clock experiments. For example, with the addition of the core-hole, the LUMO resonance moves into the electronic gap of the substrate, quenching charge-transfer processes. The effect of temperature also turns out to be important to find good agreement between theory and experiment. The LUMO +2 channel, that dominates the experimental spectra, shows a significantly enhanced coupling to the substrate for configurations far from the equilibrium structure. This results in a computed lifetime of about 6 fs at room temperature in agreement with the experimental observation -a result that cannot be reproduced by only considering the relaxed structure. The effect of temperature is much smaller on the LUMO and LUMO+1 resonances that keep weakly coupled to the substrate.

The presented cumulative spectra can be described by Voigt-type profiles obtained by convoluting a Lorentzian peak describing the elec- 
tronic resonance with a Gaussian distribution modeling the temperature broadening. However, we found that in order to get a good description it is crucial to account for the energy dependence of the statistically averaged electronic widths. This energy dependence is dominated by the variation of the density of acceptor states in the substrate. Since the density of states in the surface is approximately time independent, this observation might be interesting in connection with the development of simplified methods to account for resonant chargetransfer at finite temperatures.

We found that the presence of a core-hole not only downshifts the energy levels, but also reduces the electron-vibrational coupling, leading to a reduction of the observed thermal broadening with respect to the ground-state. Due to the core-hole attraction the molecular states are more localized on the adsorbate and appear to be less prone to the average fluctuations with respect to the environment. These observations are relevant for the interpretations of core-hole spectroscopy data. In general, a corehole strongly disturbs the electronic structure of the molecule, which might pose limits to the applicability of X-rays for the assessment of devices operating under lower energy excitations like dye-sensitized solar cells.

A sampling of static images as in the current study may fail for slower electron transfer processes, which are significantly affected by the atomic motion. Our strategy is well-placed in the range of fast processes, where electron transfer takes place while virtually no change in the underlying ionic configurations occurs. In this adiabatic limit, a detailed model of the instantaneous configuration and the electronic excitation is instrumental. A statistical treatment of available configurations remains inevitable at finite temperature.

$\mathrm{X}$-ray techniques with increasing time resolution, such as current developments in connection with free electron lasers, ${ }^{51-53}$ open new roads for probing ever faster electron dynamics on surfaces and theoretical models are required for their interpretation. While our study contributes along this line, the extraction of lifetimes in the attosecond domain requires addi- tional considerations. Since strongly coupled systems frequently do not exhibit discernible resonance features, alternative theoretical approaches like the explicit propagation of wave packets in real time are necessary to study the dynamics of such ultrafast processes. The explicit inclusion of the external exciting field in simulations will be of increasing importance in this limit.

Acknowledgement The authors acknowledge financial support from the European Union Seventh Framework Programme under the grant agreement No. 607232 (THINFACE), the Spanish Ministerio de Economia y Competitividad (MINECO) (Grant No. MAT201678293-C6-4-R), the Basque Dep. de Educación and the UPV/EHU (Grant No. IT-756-13). The work was performed within the PCAM European doctorate.

Supporting Information Available: The Supporting Information describes in detail the following points: Matching procedure of the fluctuating interface to the fixed bulk geometry. Approximate statistical independence in Fermi's golden rule. Effective Voigt-like profiles. Role of the lowest vibrational modes. Effect of using different charges for the core-hole in the pseudopotential. Molecular orbitals associated with the resonances. This material is available free of charge via the Internet at http://pubs.acs.org/.

\section{References}

(1) Young, K. J.; Martini, L. A.; Milot, R. L.; Snoeberger, R. C.; Batista, V. S.; Schmuttenmaer, C. A.; Crabtree, R. H.; Brudvig, G. W. Light-driven water oxidation for solar fuels. Coordination Chemistry Reviews 2012, 256, 2503-2520.

(2) O'Regan, B.; Grätzel, M. A low-cost, high-efficiency solar cell based on dyesensitized colloidal $\mathrm{TiO} 2$ films. Nature 1991, 353, 737-740.

(3) Grätzel, M. Dye-sensitized solar cells. Journal of Photochemistry and Photobiol- 
ogy C: Photochemistry Reviews 2003, 4, 145-153.

(4) Hagfeldt, A.; Boschloo, G.; Sun, L.; Kloo, L.; Pettersson, H. Dye-Sensitized Solar Cells. Chemical Reviews 2010, 110, 6595-6663.

(5) Kojima, A.; Teshima, K.; Shirai, Y.; Miyasaka, T. Organometal Halide Perovskites as Visible-Light Sensitizers for Photovoltaic Cells. Journal of the American Chemical Society 2009, 131, 60506051.

(6) Calió, L.; Kazim, S.; Grätzel, M.; Ahmad, S. Hole-Transport Materials for Perovskite Solar Cells. Angewandte Chemie International Edition 2016, 55, 1452214545 .

(7) Nazeeruddin, M. K.; De Angelis, F.; Fantacci, S.; Selloni, A.; Viscardi, G.; Liska, P.; Ito, S.; Takeru, B.; Grätzel, M. Combined Experimental and DFTTDDFT Computational Study of Photoelectrochemical Cell Ruthenium Sensitizers. Journal of the American Chemical Society 2005, 127, 16835-16847.

(8) Schnadt, J.; O'Shea, J. N.; Patthey, L.; Kjeldgaard, L.; Ahlund, J.; Nilson, K.; Schiessling, J.; Krempasky, J.; Shi, M.; Karis, O. et al. Excited-state charge transfer dynamics in systems of aromatic adsorbates on $\mathrm{TiO}_{2}$ studied with resonant core techniques. The Journal of Chemical Physics 2003, 119, 12462.

(9) Schnadt, J.; O'Shea, J. N.; Patthey, L.; Krempaský, J.; Mårtensson, N.; Brühwiler, P. A. Alignment of valence photoemission, x-ray absorption, and substrate density of states for an adsorbate on a semiconductor surface. Physical Review B 2003, 67, 235420.

(10) Schnadt, J.; O'Shea, J.; Patthey, L.; Schiessling, J.; Krempaský, J.; Shi, M.; Mårtensson, N.; Brühwiler, P. Structural study of adsorption of isonicotinic acid and related molecules on rutile $\mathrm{TiO} 2(110)$ II: XPS. Surface Science 2003, 544, 7486.

(11) Schnadt, J.; Schiessling, J.; O'Shea, J.; Gray, S.; Patthey, L.; Johansson, M.J.; Shi, M.; Krempaský, J.; Åhlund, J.; Karlsson, P. et al. Structural study of adsorption of isonicotinic acid and related molecules on rutile $\mathrm{TiO} 2(110)$ I: XAS and STM. Surface Science 2003, 540, 39-54.

(12) O'Shea, J. N.; Schnadt, J.; Brühwiler, P. A.; Hillesheimer, H.; Mårtensson, N.; Patthey, L.; Krempasky, J.; Wang, C.; Luo, Y.; Ågren, H. Hydrogen-Bond Induced Surface CoreLevel Shift in Isonicotinic Acid. The Journal of Physical Chemistry B 2001, 105, 1917-1920.

(13) Wurth, W.; Menzel, D. Ultrafast electron dynamics at surfaces probed by resonant Auger spectroscopy. Chemical Physics 2000, 251, 141-149.

(14) Brühwiler, P. A.; Karis, O.; Mårtensson, N. Charge-transfer dynamics studied using resonant core spectroscopies. Reviews of Modern Physics 2002, 74, 703-740.

(15) Menzel, D. Ultrafast charge transfer at surfaces accessed by core electron spectroscopies. Chemical Society reviews 2008, 37, 2212-23.

(16) Föhlisch, A.; Feulner, P.; Hennies, F.; Fink, A.; Menzel, D.; Sanchez-Portal, D.; Echenique, P. M.; Wurth, W. Direct observation of electron dynamics in the attosecond domain. Nature 2005, 436, 373-376.

(17) Jones, D. R.; Troisi, A. A method to rapidly predict the charge injection rate in dye sensitized solar cells. Physical chemistry chemical physics : PCCP 2010, 12, 4625-34.

(18) Sánchez-Portal, D. Slab calculations and Green's function recursive methods combined to study the electronic structure of 
surfaces: application to $\mathrm{Cu}(111)-(4 \times 4)$ Na. Progress in Surface Science 2007, 82, 313-335.

(19) Fratesi, G.; Motta, C.; Trioni, M. I.; Brivio, G. P.; Sánchez-Portal, D. Resonant Lifetime of Core-Excited Organic Adsorbates from First Principles. The Journal of Physical Chemistry C 2014, 118, 87758782.

(20) Martsinovich, N.; Troisi, A. Theoretical studies of dye-sensitised solar cells: from electronic structure to elementary processes. Energy \& Environmental Science 2011, 4, 4473.

(21) Prezhdo, O. V.; Rossky, P. J. Mean-field molecular dynamics with surface hopping. The Journal of Chemical Physics 1997, $10 \%, 825$.

(22) Craig, C. F.; Duncan, W. R.; Prezhdo, O. V. Trajectory Surface Hopping in the Time-Dependent KohnSham Approach for Electron-Nuclear Dynamics. Physical Review Letters 2005, 95, 163001.

(23) Akimov, A. V.; Prezhdo, O. V. The PYXAID Program for Non-Adiabatic Molecular Dynamics in Condensed Matter Systems. Journal of Chemical Theory and Computation 2013, 9, 4959-4972.

(24) HammesSchiffer, S.; Tully, J. C. Proton transfer in solution: Molecular dynamics with quantum transitions. The Journal of Chemical Physics 1994, 101, 4657-4667.

(25) Stier, W.; Prezhdo, O. V. Nonadiabatic Molecular Dynamics Simulation of LightInduced Electron Transfer from an Anchored Molecular Electron Donor to a Semiconductor Acceptor. Journal of Physical Chemistry B 2002, 106, 8047-8054.

(26) Rego, L. G. C.; Batista, V. S. Quantum Dynamics Simulations of Interfacial Electron Transfer in Sensitized TiO2 Semiconductors. Journal of the American Chemical Society 2003, 125, 7989-7997.
(27) Abuabara, S. G.; Rego, L. G. C.; Batista, V. S. Influence of Thermal Fluctuations on Interfacial Electron Transfer in Functionalized $\mathrm{TiO} 2$ Semiconductors. Journal of the American Chemical Society 2005, 127, 18234-18242.

(28) Wang, H.; Thoss, M. Multilayer formulation of the multiconfiguration timedependent Hartree theory. The Journal of Chemical Physics 2003, 119, 1289-1299.

(29) Thoss, M.; Kondov, I.; Wang, H. Theoretical study of ultrafast heterogeneous electron transfer reactions at dyesemiconductor interfaces. Chemical Physics 2004, 304, 169-181.

(30) Kondov, I.; Č́žžek, M.; Benesch, C.; Wang, H.; Thoss, M. Quantum Dynamics of Photoinduced Electron-Transfer Reactions in Dye-Semiconductor Systems: First-Principles Description and Application to Coumarin 343- $\mathrm{TiO}_{2}$. The Journal of Physical Chemistry $C$ 2007, 111, 11970-11981.

(31) Li, J.; Kondov, I.; Wang, H.; Thoss, M. Quantum dynamical simulation of photoinduced electron transfer processes in dyesemiconductor systems: theory and application to coumarin 343 at $\mathrm{TiO}_{2}$. Journal of Physics: Condensed Matter 2015, 27, 134202.

(32) Lin, H.; Fratesi, G.; Selçuk, S.; Brivio, G. P.; Selloni, A. Effects of Thermal Fluctuations on the Structure, Level Alignment, and Absorption Spectrum of Dye-Sensitized $\mathrm{TiO}_{2}: \mathrm{A}$ Comparative Study of Catechol and Isonicotinic Acid on the Anatase(101) and Rutile(110) Surfaces. The Journal of Physical Chemistry $C$ 2016, 120, 3899-3905.

(33) Soler, J. M.; Artacho, E.; Gale, J. D.; García, A.; Junquera, J.; Ordejón, P.; Sánchez-Portal, D. The SIESTA method 
for ab initio order- $\mathrm{N}$ materials simulation. Journal of Physics: Condensed Matter 2002, 14, 2745.

(34) Junquera, J.; Paz, Ó.; Sánchez-Portal, D.; Artacho, E. Numerical atomic orbitals for linear-scaling calculations. Physical Review B 2001, 64, 235111.

(35) Perdew, J. P.; Burke, K.; Ernzerhof, M. Generalized Gradient Approximation Made Simple. Physical Review Letters 1996, 77, 3865-3868.

(36) Troullier, N.; Martins, J. L. Efficient pseudopotentials for plane-wave calculations. Physical Review B 1991, 43, 1993-2006.

(37) Inglesfield, J. E. A method of embedding. Journal of Physics C: Solid State Physics 1981, 14, 3795-3806.

(38) Benesh, G. A.; Inglesfield, J. E. An embedding approach for surface calculations. Journal of Physics C: Solid State Physics 1984, 17, 1595-1606.

(39) Brivio, G. P.; Trioni, M. I. The adiabatic moleculemetal surface interaction: Theoretical approaches. Reviews of Modern Physics 1999, 71, 231-265.

(40) Brandbyge, M.; Mozos, J.-L.; Ordejón, P.; Taylor, J.; Stokbro, K. Density-functional method for nonequilibrium electron transport. Physical Review B 2002, 65, 165401.

(41) Kleinman, L. Comment on the average potential of a Wigner solid. Physical Review $B$ 1981, 24, 7412-7414.

(42) Junquera, J.; Zimmer, M.; Ordejón, P.; Ghosez, P. First-principles calculation of the band offset at $\mathrm{BaO} / \mathrm{BaTiO}_{3}$ and $\mathrm{SrO} / \mathrm{SrTiO}_{3}$ interfaces. Physical Review $B$ 2003, 67, 155327.

(43) Junquera, J.; Cohen, M. H.; Rabe, K. M. Nanoscale smoothing and the analysis of interfacial charge and dipolar densities. Journal of Physics: Condensed Matter 2007, 19, 213203.
(44) Sánchez-Portal, D.; Menzel, D.; Echenique, P. M. First-principles calculation of charge transfer at surfaces: The case of core-excited $\operatorname{Ar}^{*}\left(2 \mathrm{p}_{3 / 2}^{-1} 4 \mathrm{~s}\right)$ on $\mathrm{Ru}(0001)$. Phys. Rev. B 2007, 76, 235406 .

(45) Cvetko, D.; Fratesi, G.; Kladnik, G.; Cossaro, A.; Brivio, G. P.; Venkataraman, L.; Morgante, A. Ultrafast electron injection into photo-excited organic molecules. Phys. Chem. Chem. Phys. 2016, 18, 22140-22145.

(46) Stier, W.; Prezhdo, O. V. Thermal effects in the ultrafast photoinduced electron transfer from a molecular donor anchored to a semiconductor acceptor. Israel Journal of Chemistry 2002, 42, 213-224.

(47) Duncan, W. R.; Prezhdo, O. V. Theoretical Studies of Photoinduced Electron Transfer in Dye-Sensitized $\mathrm{TiO}_{2}$. Annual Review of Physical Chemistry 2007, 58, 143-184.

(48) Duncan, W. R.; Stier, W. M.; Prezhdo, O. V. Ab Initio Nonadiabatic Molecular Dynamics of the Ultrafast Electron Injection across the Alizarin $\mathrm{TiO}_{2}$ Interface. Journal of the American Chemical Society 2005, 127, 7941-7951.

(49) Maggio, E.; Martsinovich, N.; Troisi, A. Evaluating Charge Recombination Rate in Dye-Sensitized Solar Cells from Electronic Structure Calculations. The Journal of Physical Chemistry $C$ 2012, 116, 7638-7649.

(50) Ida, T.; Ando, M.; Toraya, H. Extended pseudo-Voigt function for approximating the Voigt profile. Journal of Applied Crystallography 2000, 33, 1311-1316.

(51) Zholents, A. A.; Zolotorev, M. S. Femtosecond X-Ray Pulses of Synchrotron Radiation. Physical Review Letters 1996, 76, 912-915. 
(52) Krausz, F.; Ivanov, M. Attosecond physics. Reviews of Modern Physics 2009, 81, 163-234.

(53) Prince, K. C.; Allaria, E.; Callegari, C.; Cucini, R.; De Ninno, G.; Di Mitri, S.; Diviacco, B.; Ferrari, E.; Finetti, P.; Gauthier, D. et al. Coherent control with a short-wavelength free-electron laser. $\mathrm{Na}$ ture Photonics 2016, 10, 176-179. 


\section{Graphical TOC Entry}

1
2

3

4

5

6

7

8

9

10

11

12

13

14

15

16

17

18

19

20

21

22

23

24

25

26

27

28

29

30

31

32

33

34

35

36

37

38

39

40

41

42

43

44

45

46

47

48

49

50

51

52

53

54

55

56

57

58

59

60

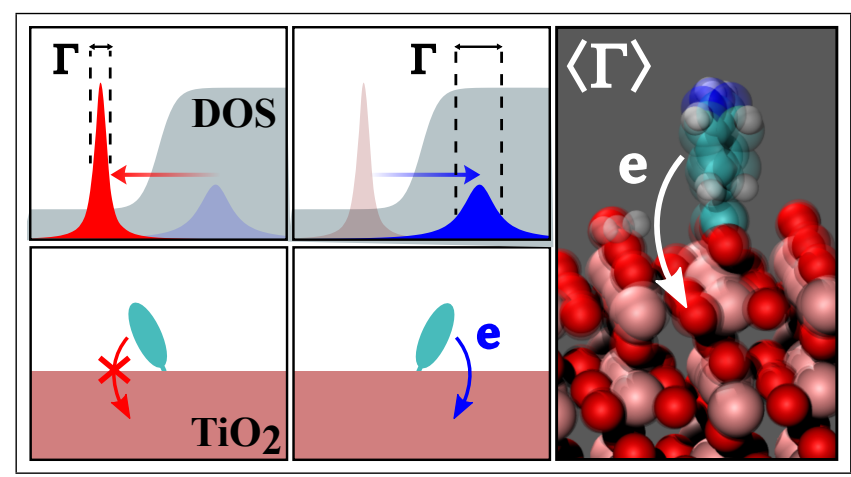

